International Journal on Artificial Intelligence Tools

Vol. 24, No. 2 (2015) 1540009 (31 pages)

(C) World Scientific Publishing Company

DOI: $10.1142 /$ S0218213015400096

\title{
Learning Relational User Profiles and Recommending Items as Their Preferences Change
}

\author{
Zaigham Faraz Siddiqui* \\ Eleftherios Tiakas ${ }^{\dagger}$ and Panagiotis Symeonidis ${ }^{\ddagger}, \S$ \\ Aristotle University of Thessaloniki, Thessaloniki, Greece \\ †tiakas@csd.auth.gr \\ †symeon@csd.auth.gr \\ Myra Spiliopoulou ${ }^{\S}$ \\ University of Magdeburg, Magdeburg, Germany \\ myra@iti.cs.uni-magdeburg.de \\ Yannis Manolopoulos \\ Aristotle University of Thessaloniki, Thessaloniki, Greece \\ manolopo@csd.auth.gr
}

Received 29 September 2014

Accepted 25 November 2014

Published 13 April 2015

\begin{abstract}
Over the last decade a vast number of businesses have developed online e-shops in the web. These online stores are supported by sophisticated systems that manage the products and record the activity of customers. There exist many research works that strive to answer the question "what items are the customers going to like" given their historical profiles. However, most of these works do not take into account the time dimension and cannot respond efficiently when data are huge. In this paper, we study the problem of recommendations in the context of multi-relational stream mining. Our algorithm "xStreams" first separates customers based on their historical data into clusters. It then employs collaborative filtering $(\mathrm{CF})$ to recommend new items to the customers based on their group similarity. To evaluate the working of xStreams, we use a multi-relational data generator for streams. We evaluate xStreams on real and synthetic datasets.
\end{abstract}

Keywords: Stream recommenders; evolving user preferences; stream mining.

*Work done while with the University of Magdeburg, Magdeburg, Germany.

$\S$ Corresponding authors 


\section{Introduction}

Data mining for recommendation engines is a mature domain, and the underpinnings of the core procedure nowadays constitute public knowledge. In the simplest scenario, the recommender matches the preferences of a peer user to the preferences of users similar to her, and then recommends items that these similar users have liked. In the most cases the user preferences are retrieved through the web, recorded in historical databases and used by the recommendation engines, which are included in internet applications, e-business systems, e-shops etc. Data mining and Web mining are responsible for learning a model on user similarity; the simplest case involves offline clustering users on similarity of preference. However, this traditional view of a recommendation engine oversees the fact that user preferences change over time: the preferences of a 22-year old girl are not the same she had when she was a teenager. In this study, we propose a method $x$ Streams that couples data stream mining with a recommendation engine to learn and exploit the evolution of user preferences. xStreams is evaluated on a real and a synthetic dataset. To generate the synthetic data, we also propose a time-evolving multi-relational data generator MultiGen, which creates evolutionary user profiles to simulate the behaviour of the real users.

The awareness on the importance of time for a recommendation engine gained momentum during the Netflix competition, which was a million-dollar competition for making a recommender that outperforms the existing Netflix system. The competition's winner, Yehuda Koren, has demonstrated how the incorporation of time into an ensemble of learners boosts predictive performance. ${ }^{10}$ Remarkably, the approach of Koren cannot adapt to the evolving preferences of people: it rather captures the preferences of the people during the time period of observation and learns a static model on them. This is not appropriate for the dynamic environment of a recommendation engine: the model learned by the miner on the basis of observed transactions must be adapted as new transactions arrive, because new transactions reflect the evolution of people's preferences.

To deal with this issue, we observe the activities of the users as a stream, and perform stream clustering upon similar entities. At each timepoint, the recommendation engine uses the clusters to identify users similar to the peer user and deliver suggestions to her. However, the task of clustering similar users on their evolving preferences does not agree with the conventional stream mining paradigm either! To juxtapose the task of learning the preferences of evolving users to a conventional stream mining task, consider following example of a traditional stream.

Assume an e-shop that records customer purchases. The purchases constitute a stream, an instance of which contains the identifiers of the customer and of the purchased product, the timepoint of the purchase, and further information such as the product's price, payment option, shipment option, delivery address etc. The shop is interested in detecting fraudulent purchases. To this purpose, transactions are clustered on similarity and outliers are inspected. As new purchases arrive, the 
clusters evolve, some transactions turn to be similar to earlier outliers and are thus suspect, while new outliers show up and must be inspected, too. In other words, the stream clustering algorithm adapts the clusters to arriving data and allows for the detection of new kinds of fraudulent transactions.

The above example describes unsupervised model learning on a stream of instances - the purchases. The recommendation scenario is a bit different though.

Assume again the e-shop that records customer purchases as a stream. The shop is interested to recommend to each customer $X$ a product that she will like, knowing that her attitude to some products may have changed. To this purpose, the list of products preferred by $X$ must be extended whenever she buys a new product, but the timepoint of the purchase must also be recorded. The miner must now cluster together users who are similar to each other because they (a) now like the same products and (b) have liked the same products in the past.

The two examples differ, because the first one involves clustering the instances on similarity, while the second one requires clustering the users on similarity of the sets of instances associated to them at different moments. This task requires multirelational stream clustering. We build upon our earlier method ${ }^{22}$ for clustering a stream of complex objects, and extend it to deliver insights on user similarity on the basis of their past transactions.

This article presents an approach for recommending items to the users as they evolve over time. The main contributions of the papers are: (1) we propose a incremental multi-relational method for recommending items to the users, (2) the proposed method is hybrid and combines similarity computation from content-based and collaborative filtering, and (3) we present a framework for evaluating recommenders over streams. The rest of the paper is organized as follows. In Section 2 we discuss related work on recommendations over dynamic data and on multi-relational stream learning. In Section 3 we present our approach for the formulation of recommendations towards users with evolving preferences. In Section 4 we summarize the multi-relational generator ${ }^{25}$ we use for synthetic set generation. In Section 5 we discuss issues and challenges that are encountered during the evaluation of streaming recommenders. In Section 6 we report on our experiments with historical data for the MovieLens and a synthetic data set. The last section concludes our work.

\section{Related Work}

In this Section, we will present the main work in collaborative filtering, contentbased filtering and hybrid recommender systems. Moreover, we will discuss the latest progress on time-aware recommender systems, which is a rather new research area. Notice that related work comes from two different direction: the domain of (i) temporal mining, and (ii) stream mining. The difference between these research directions is that the former considers time as an additional dimension and learns model on the complete data, while for the latter data elements are discarded or archived based on age and the model is learned only over most recent data. These models are adaptable and get updated when concept drift occurs. 


\subsection{Content-based, collaborative filtering and hybrid recommenders}

In the context of recommender systems, there exist three parallel approaches: collaborative filtering (CF), content-based filtering $(\mathrm{CB})$ and hybrid methods.

Collaborative filtering algorithms recommend those items to the target user, that have been rated highly by other users with similar preferences and tastes. ${ }^{19,21,7,12}$ In most CF approaches, only the item and users' identifiers are accessible and no additional information over items or users is provided. Websites that provide recommendations in the form, "Customers who bought item $i$ also bought item $y$ ", typically fall under collaborative filtering approaches. Grouplens research group ${ }^{19}$ introduced a collaborative filtering algorithm, known as user-based CF, because it employs users' similarities for the formation of the neighbourhood of nearest users. Another CF algorithm proposed by Sarwar et al. ${ }^{21}$ is known as item-based CF algorithm, because it employs items' similarities for the formation of the neighbourhood of nearest users. A pitfall of CF is the cold start problem: new items have received only few ratings, so they cannot be recommended; new users have performed only few transactions, so there are hardly other users similar to them.

Herlocker et $a .^{7}$ proposed a variation of the previous algorithms, denoted as Weighted Similarity (WS). It weights similarities by the number of common ratings between users/items. In particular, if sim is a similarity measure, e.g. Pearson or cosine, then WS is equal to $\frac{\max (c, \gamma)}{\gamma} \cdot \operatorname{sim}$, where $c$ is the number of co-rated items. User-based CF takes into account only the set of items, that are co-rated by both users. This, however, ignores the items rated by only one of the two users. The number of the latter items denotes how much their preferences differ. Especially for the case of sparse data, by ignoring these items we discard significant information. Deshpande and Karypis ${ }^{2}$ apply item-based CF algorithm combined with conditional-based probability similarity and Cosine Similarity. Xue et al. ${ }^{28}$ suggest a hybrid integration of aforementioned memory-based algorithms with model-based algorithms.

Content-based filtering assumes that each user operates independently. It exploits information derived only from documents or item features, e.g. terms or attributes. ${ }^{18,17,13}$ In particular, it exploits a set of attributes which describes the items and recommend other items similar to those that exist in the user's profile. In this way, the cold start problems for new items and new users are alleviated, provided that users prefer items that are similar in content to those they have already chosen. However, the pitfall of CB is that there is no diversity in the recommendations. That is, the user gets recommendations that are very familiar to her, since these recommended items are similar to those in her item profile.

There have been several hybrid attempts to combine $\mathrm{CB}$ with $\mathrm{CF}$. The Fab System, ${ }^{1}$ combines $\mathrm{CB}$ and $\mathrm{CF}$ in its recommendations, by measuring similarity between users after first computing a profile for each user. Fab initially categorizes documents by a CB filter and then recommends them to the user based on her 
relevance feedback. In contrast, the CinemaScreen System $^{20}$ runs CB on the results of CF. In particular, CinemaScreen system computes predicted rating values for movies based on $\mathrm{CF}$ and then applies $\mathrm{CB}$ to generate the recommendation list. Our approach can be categorized as a hybrid method, since it combines both contentbased characteristics and collaborative filtering. In contrast to the aforementioned hybrid methods, we also incorporate in our model the time dimension.

Finally, apart from blending the content with rating data, Social web has allowed the emergence of new data combinations that can provide even more robust recommendations. ${ }^{16}$ For instance, social networks such as Facebook, LinkedIn, etc., include information about the connections between humans. There are two main types of recommendations in social networks. The first one is related to the link prediction task, whereas the second one refers to the rating prediction and item recommendation task.

\subsection{Time-aware recommender systems}

In the last years, there are scholars who proposed methods that attempt to capture the temporal aspects of user behaviour, while others investigated the updating of recommenders to change in the user behaviour. Remarkably, these two categories of methods are distinct, in the sense that studies capturing temporal aspects produce static models, while studies capturing change produce dynamic models. Our approach belongs to the second category. In this Section, we will briefly discuss the approaches of Ding and Li, ${ }^{5}$ Yuan et al. ${ }^{29}$ Xiang et al. ${ }^{27}$ and Yehuda Koren,,${ }^{9,10}$ which belong to the first category.

Ding and $\mathrm{Li}^{5}$ proposed a method that assigns time weights for purchases of items by decreasing the weight to old purchasing data. They have shown that the users' purchase habits vary and even the same user has quite different preferences towards the same items over time.

Xiang et $a .^{27}$ proposed a framework that models users' long-term and shortterm product preferences of a user over time. Their model is built on a Session-based Temporal Graph (STG), which incorporates user, products and session information. Based on the aforementioned STG graph, the user-product bipartite graph denotes the long term preferences of a user, whereas the product-session bipartite graph denotes the short term preferences of a user. Moreover, Xiang et al. ${ }^{27}$ proposed also a novel recommendation algorithm named Injected Preference Fusion (IPF) and extended the personalized Random Walk for temporal-based product recommendation. IPF starts from the user node and propagates to products rated or bought by the user at a previous session, and then tends to propagate to unknown products similar to user's long-term preferences; while preferences injected into the session nodes will propagate to products visited by the user at a past session, and then tend to propagate to unknown products similar to user's short-term preferences.

Yehuda Koren ${ }^{9,10}$ considered the scenario of item rating by capturing the influence of time and has identified the following aspects: (i) user-bias changes over 
time, i.e. the user's rating assigned to an item may vary over time and may exhibit periodicity, (ii) the item-bias changes over time, and (iii) the ratings submitted by a given user may vary depending on time of day, day of week or period in the year. Based on the aforementioned aspects a baseline predictor (which assigns to each item an average rating $\mu$ ) can be extended as shown in Eq. (1): ${ }^{9,10}$

$$
\widehat{r}_{u i}=\mu+b_{u}\left(t_{u i}\right)+b_{i}\left(t_{u i}\right)
$$

where $\widehat{r}_{u i}$ is the predicted rating of a user $u$ for an item $i, \mu$ is the overall average rating, $b_{u}$ and $b_{i}$ are the user and item bias over $\mu$ respectively, and $t_{u i}$ denotes the rating of user $u$ on item $i$ at day $t$. The above baseline predictor can be easily integrated into a factor model. ${ }^{9,10}$ Koren and Bell ${ }^{11}$ proposed timeSVD++, a set of predictors that learn latent factors thereby exploiting implicit information on user preferences, i.e. which items users rate, regardless of their rating, and the impact of time, including day effects on a user's rating attitude. timeSVD++ was shown to offer accuracy superior to SVD++. ${ }^{11}$

Yuan et al. ${ }^{29}$ exploited temporal characteristics of users' check-in information to provide location recommendations. In particular, they split time in multiple slots. Then, they fill these slots with check-in values that users made at each specific hour of the day. Moreover, they use a User-Time-Location (UTL) cube to present check-in records. An element $C_{u, t, l}$ of UTL cube, denotes a user $u$, who visited a location $l$ at time slot $t$. They incorporate temporal dimension in their model, by predicting the probability that a user $u$ will check-in a location $l$ at a specific time $t$, as shown by Eq. (2):

$$
\widehat{C_{u, t, l}^{(t)}}=\frac{\sum_{v} w_{v, t}^{(t)} * C_{v, t, l}}{\sum_{v} w_{v, t}^{(t)}}
$$

where, $w_{v, t}^{(t)}$ is the temporal behavior similarity between users $u$ and $v .^{29}$

All the above approaches are temporal and of evolutionary nature, and they are not appropriate for stream mining, because stream mining requires that a model is adapted to new data as they arrive, while the aforementioned methods learn a model that explains all data seen thus far. An excellent elaboration on this issue has been written down by Koren and Bell themselves, ${ }^{11}$ subsection 5.3.4.1 "Predicting future days" (page 160), stating among others that "... for those future (untrained) dates, the day-specific parameters should take their default value." Although they refer explicitly to day-specific parameters, we must keep in mind that all parameters of the timeSVD ++ have been specified with cross validation upon the whole dataset. ${ }^{11}$ Once new untrained data arrive, cross validation must be rerun, because, as Koren and Bell state on the same page 160 "... our temporal modelling makes no attempt to capture future changes. All it is trying to do is to capture transient temporal effects, which had a significant influence on past user feedback", whereby we would like to stress the word "past". Another way of formulating the statements of Koren and Bell is that timeSVD++ learns a static model over a finite dataset, and can 
be applied on future stream data if and only if all thinkable concept drift can be captured by modelling this finite dataset, i.e. if and only if future data follow exactly the same distribution as past data. Stream mining research encompasses methods for the analysis of data for which this assumption does not hold.

\subsection{Stream mining for recommender systems}

The importance of model updating in a recommender has been demonstrated by Dias et $a l .{ }^{3}$ they have experimentally shown that updating the checkout recommender model files consistently resulted in an increase in the number of new shoppers using the recommender system.

A truly adaptive recommendation engine for streams has been proposed by Nasraoui et $a l .{ }^{15}$ for the prediction of the next inspected page in user sessions. The stream under inspection is the clickstream, in which sub-sessions are observed, matched to already seen complete sessions, from which the top-N recommendations are derived. The recommendations are formulated and evaluated immediately by reading in the users' responses, essentially, the next page click per session and incorporating them into the model learned thus far. For model learning and updating, Nasraoui et al. consider $\mathrm{kNN}$, which finds the $\mathrm{k}$ most similar sessions to an input sub-session, and their stream clustering algorithm TECNO-STREAMS, ${ }^{14}$ which learns and adapts profiles (as abstractions of sessions) and returns for each input sub-session the profile most similar to it. ${ }^{15}$

Nasraoui et al. ${ }^{15}$ have tested their adaptive recommender on two Web clickstreams, thereby simulating two evolution scenarios. In the "induced drastic sequential user profile evolution" (scenario D), the sessions of the data set are clustered into profiles, and the sessions of each profile are delivered to the recommender one profile at a time. Hence, once the session of a given profile are read through, there come sessions that do not fit to the model learned by the recommender at all - this corresponds to a shift. In the "natural or mild chronological order" (scenario M), the sessions arrive in chronological order, hence the profiles are mixed. The evaluation on moving average of the F-measure shows that $\mathrm{kNN}$ is of slight advantage over TECNO-STREAMS in the scenario D, because the former relies more on model learning, while the two algorithms behave similarly under Scenario M.

Our approach follows the same philosophy as the recommender of Nasraoui et $a l .{ }^{15}$ : we anticipate that the recommender should forget old instances and learn from newly arriving instances immediately; the recommender uses profiles instead of matching individuals; the profiles are adapted over time to respond to evolving user preferences. Our evaluation is also dictated by the idea that the recommender must deal with both sudden shift (as in scenario D) and with arbitrary drift (scenario M). ${ }^{\text {a }}$ In short, we share the core ideas of proper stream learning under drift.

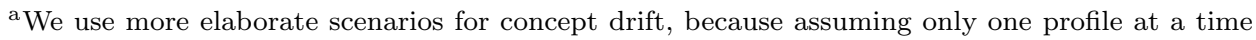
and abrupt change, as in scenario $\mathrm{D}^{15}$ is a bit simplistic. 
Yet, the objective of Nasraoui et al. ${ }^{15}$ makes their algorithm not comparable to ours. In particular, their recommender predicts the next page of a user session, and adapts as more and more of the session is observed. Although it is possible to express the sequence of items ever observed by a user as a session, this is not desirable: a user session is a matter of moments or hours, while the interaction of a user with an e-shop may extend arbitrarily across time; forgetting the beginning of a session as the session progresses is unintuitive, while forgetting very old purchases of a user is a reasonable option; a user revisits pages $\mathrm{s} /$ he has studied before, thus allowing for forms of sub-session matching that make no sense when comparing the preferences of users. Most importantly, a session in clickstream mining is a sequence of page identifiers, whereby we incorporate into user similarity and model learning also the properties of the products observed at each moment, allowing the impact of old products to fade out. A comparison of clustering algorithms might have been possible, but the experiments of Ref. 15 have shown that $\mathrm{kNN}$ is mostly superior and never truly inferior to TECNO-STREAMS. Hence, we compare our approach to an adaptive collaborative filtering stream recommender that essentially uses kNN.

Recently, Diaz-Aviles et al. have proposed Stream Ranking Matrix Factorization $(\mathrm{RMFX}),{ }^{4}$ an algorithm that is intended to perform matrix factorization and item ranking on a stream. The focus of the algorithm is on maintaining an up-to-date model on the basis of possibly small, intelligently devised samples. Accordingly, the experimentation was done on two time slots only, where one slot used for learning, the other for testing. It delivered insights on the efficiency of the algorithm but not on its adaptivity. Beyond this, RMFX must know the sets of users and of items in advance similarly to timeSVD++; then, it can fill it gradually. This makes the algorithm inappropriate for our scenario: in a realistic long-term setting, new customers show up and new items may be put to sale at any time, hence the matrix dimensions cannot be known in advance. Note that the previously mentioned algorithm of Nasraoui et al. ${ }^{15}$ has neither caveat: it has been designed to be adaptive and it does not need to know all users nor all pages that a user may choose to access. Hence, in our evaluation we use as baseline an algorithm that satisfies the same core properties as the one of Ref. 15 .

\section{The xStreams Method}

Our stream recommender xStreams consists of two modules. (i) The back end is an incremental, adaptive learner that processes the streams of activities and associates it with earlier obtained and updated information on the entities involved - users/customers and items/products. (ii) The front end builds on top of the incremental learner to deliver the top- $N$ items as recommenders to each user. We first describe the process of reading instances from the stream of postings and combining them with earlier recorded information on the referenced entities (users, items). This process is called "incremental propositionalisation" and comes 
(a) Original Schema

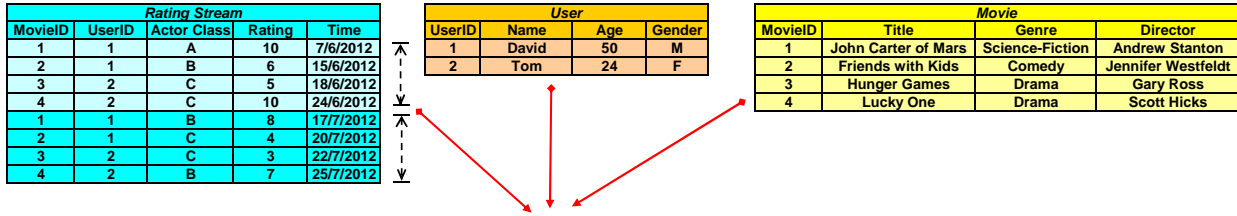

(b) Natural Join

\begin{tabular}{|c|c|c|c|c|c|c|c|c|c|c|}
\hline MovielD & Title & Genre & Director & UserID & Name & Age & Gender & Actor Class & Rating & Time \\
\hline 1 & John Carter of Mars & \begin{tabular}{|l|} 
Science-Fiction \\
\end{tabular} & Andrew Stanton & 1 & David & 50 & $M$ & $\mathrm{~A}$ & 10 & $7 / 6 / 2012$ \\
\hline 2 & Friends with Kids & Comedy & Jennifer Westfeldt & 1 & David & 50 & $\mathrm{M}$ & B & $\frac{26}{6}$ & $15 / 6 / 2012$ \\
\hline 3 & Hunger Games & Drama & Gary Ross & 2 & Tom & 24 & $F$ & c & 5 & $18 / 6 / 2012$ \\
\hline 4 & Lucky One & Drama & Scott Hicks & 2 & Tom & 24 & $F$ & c & 10 & $24 / 6 / 2012$ \\
\hline 1 & John Carter of Mars & Science-Fiction & Andrew Stanton & 1 & David & 50 & M & B & 8 & $17 / 7 / 2012$ \\
\hline 2 & Friends with Kids & Comedy & Jennifer Westfeldt & 1 & David & 50 & M & C & 4 & $20 / 7 / 2012$ \\
\hline 3 & Hunger Games & Drama & Gary Ross & 2 & Tom & 24 & $\mathbf{F}$ & C & 3 & $22 / 7 / 2012$ \\
\hline 4 & Lucky One & Drama & Scott Hicks & 2 & Tom & 24 & $\mathbf{F}$ & B & 7 & $25 / 7 / 2012$ \\
\hline
\end{tabular}

(c) Propositionalised Table

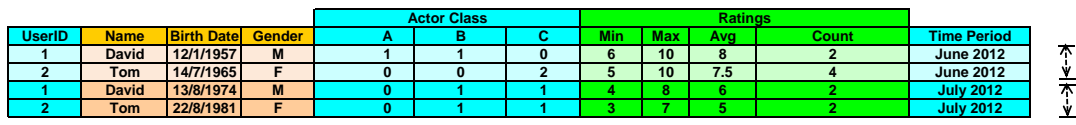

Fig. 1. User and Movie entities linked to rating entities: (a) the original schema consists of three tables, all of which are actually streams; (b) the natural join over them results in one entry/vector per rating, while (c) the propositionalisation operation produces one vector per user. We perform incremental propositionalisation to learn over the User entities, as they grow with information on ratings and movies.

from our earlier work. ${ }^{22}$ We use the results of this process to compute the similarity of a given user to other users, as described next in subsection 3.2. The back end and the front end algorithms are presented as pseudo-code in subsection 3.3, where we also discuss their complexity. As running example we use the multi-table stream of ratings, users and items in Figure 1.

\subsection{Incrementally combining information on users, items and ratings}

The core source of information for the recommendation engines is the stream of activities performed by the users. In the classical stream mining scenario, stream instances are observed, processed and forgotten. In the context of learning, a stream record is used to adapt the model and is then forgotten. However, for the recommendation scenario we study, we want to learn and adapt a model of the users, so that the recommender can respond to changes in a user's ratings towards items.

\subsubsection{Learning task on multiple streams}

In Figure 1(a), we depict users and their ratings for movies by means of three tables. Model learning and updating by the recommender's back end will be performed on the table User, which is linked to the table Rating, which is in turn linked to the table Movie. The stationary information on users, such as name and gender, is stored in the table User, but learning must also exploit each user's ratings for movies, as 
well as the properties of the movies themselves, e.g. genre. Since ratings arrive at any time, Rating is a stream, the records of which are seen and forgotten. In contrast, users and movies are perennial entities: perennial entities cannot be forgotten as they may reappear again. They are stored in the database when inactive and get retrieved from it when new ratings arrive for them. Nonetheless, new users and new movies may also arrive at any time, hence User and Movie are also streams streams of perennial entities. ${ }^{24}$ For perennial entities, we use the terms "table" and "stream" interchangeably hereafter, while for a ephemeral records like the ratings we use solely the term "stream".

The back-end of our method, xStreams_BackEnd, is an adaptive stream mining algorithm that learns a model over the table User — as it is extended with information from the streams Rating and Movie. However, the natural join of entities of these entity types is not appropriate for learning: as can be seen in Figure 1(b), this join result contains as many user entities as ratings - user David appears as four independent entities. For model learning, we rather need an expansion of table User with the data from the other tables. To this purpose, we use the incremental propositionalisation algorithm proposed in Ref. 22: it expands the so-called "target stream" (here: User) with information from the other streams, and produces one entry per entity of the target, as can be seen in the first two rows of the propositionalised table in Figure 1(c). When new stream records arrive, the vectors of the entities are updated; in Figure 1(c), we see the entries/vectors of David and Tom for June 2012 and then for July 2012. ${ }^{\text {b }}$

\subsubsection{Incremental propositionalisation for learning}

More formally, let $\mathcal{T}$ the target stream, i.e. the stream, on which we want to perform the learning: in our recommendation scenario, $\mathcal{T}$ is the stream of users (cf. User in Figure 1). It is a stream, because new users arrive at any time. It is the target stream, because we want to learn the user profiles and exploit them to compute user similarity. For this learning task, we combine $\mathcal{T}$ with information from further streams $\mathcal{T}_{1}, \ldots, \mathcal{T}_{J}$ referencing it $^{\mathrm{c}}$; in our recommendation scenario, these further streams are the stream of ratings and the items (also a stream) — cf. Rating, Movie in Figure 1. We slide a window of length $W$ timepoints over the streams, so that the data observed at timepoint $t$ are the entities from all streams observed in the interval $(t-W, t]$. Entities outside this interval are forgotten.

Let $\operatorname{schema}(X)$ be the schema of any of these streams $X \in \mathcal{A}:=\left\{\mathcal{T}, \mathcal{T}_{1}, \ldots, \mathcal{T}_{J}\right\}$. At each timepoint $t$, our incremental propositionalisation method ${ }^{22}$ expands each

\footnotetext{
${ }^{\mathrm{b}}$ In Figure 1(b), we show each user twice to demonstrate the differences in the aggregated ratings between June and July. The learner will see only one entry/vector per user: in June 2012 it uses the June vector, in the next month this old vector is replaced by the July vector.

${ }^{\mathrm{c}}$ Any of $\mathcal{T}_{x}$ may be a static table, but we consider the general case where all of them are streams. For example, if movie genre were not an attribute but rather an entity type Genre, then we could assume that all possible genres are known in advance, hence Genre would be a table rather than a stream.
} 
entity $u \in \mathcal{T}$ with the contents of the entities that reference $u$ - they constitute the set matches $(u)$, the elements of which belong to different streams from $\mathcal{A}$. For this expansion operation, our method extends $\operatorname{schema}(\mathcal{T})$ by turning the values of the elements in matches $(u)$ into new columns/features for $e_{u}$. The set of features thus generated can change at each timepoint, we therefore denote it as Features $(t)$. In particular, at timepoint 0 :

- For each numerical attribute $A$ in $\cup_{X \in \mathcal{A}} \operatorname{schema}(X)$ that appears in the schema of some element $y \in$ matches $(u)$, we add to Features(0) four features: the min, max, average and count for $A$, and we store in them the corresponding values seen in $y$. In our running example, we have calculated the min, max, average and count of ratings for each user_id per month - see Figure 1(c).

- For each nominal attribute $A$ in $\cup_{X \in \mathcal{A}} \operatorname{schema}(X)$, we create $r_{A}$ features, one per distinct value of $A$ observed at timepoint 0 for all entities in the target stream. For an entity $x$, each of those features takes the value 1 (one) if the original attribute value was in an entity in matches $(x)$ and 0 (zero) otherwise.

At a later timepoint $t$, we update the numerical features by adding values for the arriving entities in matches $(x)$ and by subtracting values for the entities that exit the sliding window, i.e. have been seen earlier than $t-W$. For the nominal attributes, we can extend Features $(t)$ as new, previously unseen, nominal values arrive. However, it is not feasible to expand Features $(t)$ to unlimited values. Rather, we set an upper threshold size $\tau$ to the number of generated features per attribute and encode the values observed thus far for this attribute into $\tau$ derived features. This encoding is based on grouping values that appear in otherwise similar entities together into $\tau$ clusters. Details on this encoding are provided in Ref. 22.

As can be seen in the two entries per user of Figure 1(c), the propositionalisation algorithm delivers at each timepoint the vector of each active user, i.e. for each user who has performed some rating inside the sliding window. The user's vector contains the information obtained on this user from the data in the window; these data are summarized, while data that have slide outside the window are forgotten. Details on window sliding and memory management can be found in Ref. 23 .

\subsection{Computing the similarity of evolving users}

The vectors of the active users form the basis for computing user-user similarity. We consider two aspects of similarity between users: similarity on the basis of summarized past preferences and similarity on the basis of current ratings. We describe these two types of similarity below, and then explain how we combine them into a single similarity function. It must be stressed that the similarity values change as new ratings arrive, hence we need to update the similarity matrix in an incremental way or replace it with some surrogate that can be computed efficiently. 


\subsubsection{Similarity on the basis of past preferences}

Let $u, v$ be two users that are active at timepoint $t$, i.e. have performed ratings in the interval $(t-W, t]$, where $W$ is the window size. Let $e_{u}^{t}$, respectively $e_{v}^{t}$ be the updated vectors of these two users after propositionalisation on all information within the interval. We define the similarity between these users with the function:

$$
\operatorname{sim} C B(t, u, v)=\frac{e_{u}^{t} \cdot v_{v}^{t}}{\left|e_{u}^{t}\right| \cdot\left|e_{v}^{t}\right|}
$$

The postfix CB in the name of $\operatorname{sim} C B()$ stands for "Content-Based" and reflects the fact that attributes of the users are also taken into consideration by the similarity measure.

For the efficient computation of similarity on the basis of past preferences, we couple incremental propositionalisation with stream clustering of the users' entries. In particular, at each timepoint $t$, we retrieve from secondary storage all users who performed ratings within the interval $(t-W, t]$, i.e. all active users. We expand the entries of these users with incremental propositionalisation, place them into $K$ clusters and then adapt the clusters through centroid re-computation. ${ }^{23}$ Then, for each user $u$ we can return the $k$ most similar users by assigning $u$ to the cluster/profile with the closest centroid and then depicting the $k$ nearest neighbours to $u$ from this cluster.

It is thinkable that the cosine similarity used in Eq. (3) is extended to consider only co-inspected items, in a similar way that only co-rated items are used in Collaborative Filtering to compute user similarity. In particular, for the similarity between two users in $\mathrm{CF}$, items not rated by one of the users are ignored. By this, it is avoided that user similarity takes into account items that one user has never inspected. In $\operatorname{sim} C B()$, we could similarly restrict the similarity computation to skip features (derived attributes, cf. subsection 3.1) that have not perceived by one of the users being compared. For example, if a user has never seen a movie of a specific producer, we could ignore the attribute referring to this producer, when comparing this user with others. This extension has not been considered in the following; it is left as future work.

\subsubsection{Similarity on the basis of ratings}

Using the underpinnings of Refs. 7 and 12, let the rating of a user $u$ over an item $i$ be denoted as $r_{u, i}$. If the user has not rated the item $i$ we set $r_{u, i}$ to NULL. Since we perform collaborative filtering on a stream, we slide a window of length $W$ over the stream of ratings and consider at each time point $t$ only the set of ratings $R_{t}$ inside the window $(t-W, t]$. At timepoint $t$, let $I_{t, u}$ be the set of items rated by $u$ within $R_{t}$ and $I_{t, v}$ the corresponding dataset for another user $v$. Then, the ratings-based similarity between $u$ and $v$ is computed as: 


$$
\operatorname{sim} C F(u, v)=\frac{\sum_{i \in I_{t, u} \cap I_{t, v}}\left(r_{u, i} \cdot r_{v, i}\right)}{\sqrt{\sum_{i \in I_{t, u}}\left(r_{u, i}\right)^{2}} \sqrt{\sum_{i \in I_{t, v}}\left(r_{v, i}\right)^{2}}}
$$

where $I_{t, u}$ and $I_{t, v}$ are computed anew at each time point $t$ and may have an empty intersection inside some window, although they were overlapping before that window.

Combining different aspects of user similarity: The conventional similarity function $\operatorname{sim} C F()$ exploits similarity of ratings between two users. Knowledge about each user's profile and expressed past preferences is captured by our new similarity function $\operatorname{sim} C B()$, which exploits accumulated past information from the aggregated feature profile of the users. As in conventional collaborative filtering, the expected rating is computed as the weighted average of the ratings made by users similar to $u$, but xStreams combines the two similarity functions when it computes the expected rating of user $u$ for item $j, \hat{r}_{u, j}$. For rating prediction, we use Eq. (5), which is explained in subsection 3.3.2.

$$
\hat{r}_{u, j}=a v g_{u}+\frac{\sum_{v \in \operatorname{Top}_{\operatorname{sers}}^{k} \wedge r_{v, j} \operatorname{NOTNULL}} \operatorname{simTotal}(t, u, v) *\left|r_{v, j}-\overline{r_{j}}\right|}{\sum_{v \in \operatorname{TopUsers}_{u}^{k}} \operatorname{simTotal}(t, u, v)} .
$$

\section{3. $x$ Streams_BackEnd and $x$ Streams_FrontEnd}

The $x$ Streams_BackEnd adaptive learner and xStreams_FrontEnd recommendation interface are decoupled, indirectly interacting modules. The back end slides a window of width $W$ over the stream and maintains the perennial entities of seen users and products in a database. It maintains the learned user profiles up to date. These are used by the front end for the identification of the $k$ most similar users to a given user $u$, for whom the algorithm formulates $n$ recommendations.

\subsection{1. xStreams_BackEnd}

The pseudo-code of our adaptive learner is depicted in Algorithm 1. At each timepoint $t$, the algorithm processes all ratings arrived in $(t-w, t]$. These ratings constitute a set $R_{t}$ (line 3 ), from which the active users $U_{\text {act }}$ in the interval are extracted. For a user $u$ that has performed ratings in $R_{t}$, her vector is fetched from the database $D$ (line 5 ). The vector is modified to accommodate the current ratings (line 6), as described in Section 3.1. For a user that already exist in $U_{\text {act }}$, the algorithm replaces the old vector with the newer one (line 8). The vectors of new users are simply inserted it into the list (line 9). All the users that have become inactive, i.e. they have no ratings in $R_{t}$, are removed from the list of active users (line 10). Since BackEnd processes a continuous stream, hence it contains no return operation. It rather writes the updated list of users to the Output (line 11) before processing the new timepoint (line 2). 


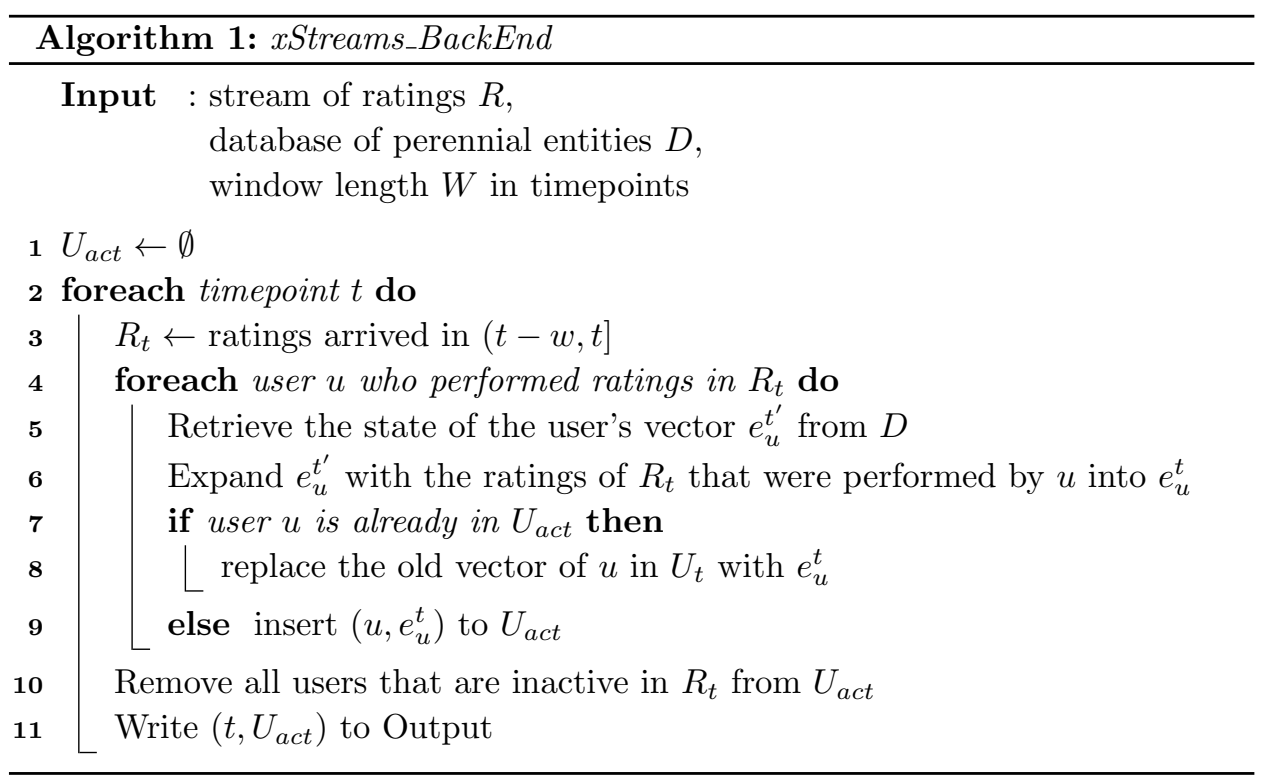

\subsection{2. xStreams_FrontEnd}

The interactive front end of our recommender predicts the items and their ratings for a specific user $u$. The pseudo-code is given in Algorithm 2. Note that the timepoint $t$ is the timepoint "now", the moment at which user $u$ is observed. It must be passed as parameter to the interface between front end and back end, so that the correct similarity values are computed on the basis of the stream chunk $R_{t}$ (cf. Algorithm 1).

To build the set TopUsers ${ }_{u}^{k}$ (line 1), we compute the similarity between $u$ and all other active users by using both $\operatorname{sim} C B()$ and $\operatorname{sim} C F()$. We use $\operatorname{sim} C B()$ of Eq. (3) to compute the similarity of $u$ to each user $u^{\prime} \in U_{\text {act }}$ (line 11, Algorithm 1), where similarity refers to aggregated information on these users. We use $\operatorname{sim} C F()$ of Eq. (4) to compute the similarity of $u$ to active users on the non-aggregated individual ratings in $R_{t}$. We then compute for each of the users in $C B_{u}(t) \cup C F_{u}(t)$ the value ${ }^{\mathrm{d}}$

$$
\operatorname{sim} \operatorname{Total}(t, u, v)=\text { weight } * \operatorname{sim} C B(t, u, v)+(1-w e i g h t) * \operatorname{sim} C F(u, v) .
$$

Thereafter, we sort the users on decreasing similarity and depict the top- $k$ users, forming the set TopUsers ${ }_{u}^{k}$.

If the weight of $\operatorname{sim} C B()$ is set to 1 , then we ignore $\operatorname{sim} C F()$. This makes sense if the current data are so volatile that we cannot draw safe conclusions from the

\footnotetext{
d This value might be perceived as the result of a similarity function $\operatorname{sim}()$. However, this operation can be computed only over the set $C B_{u}(t) \cup C F_{u}(t)$ for some user $u$; these sets are computed independently.
} 


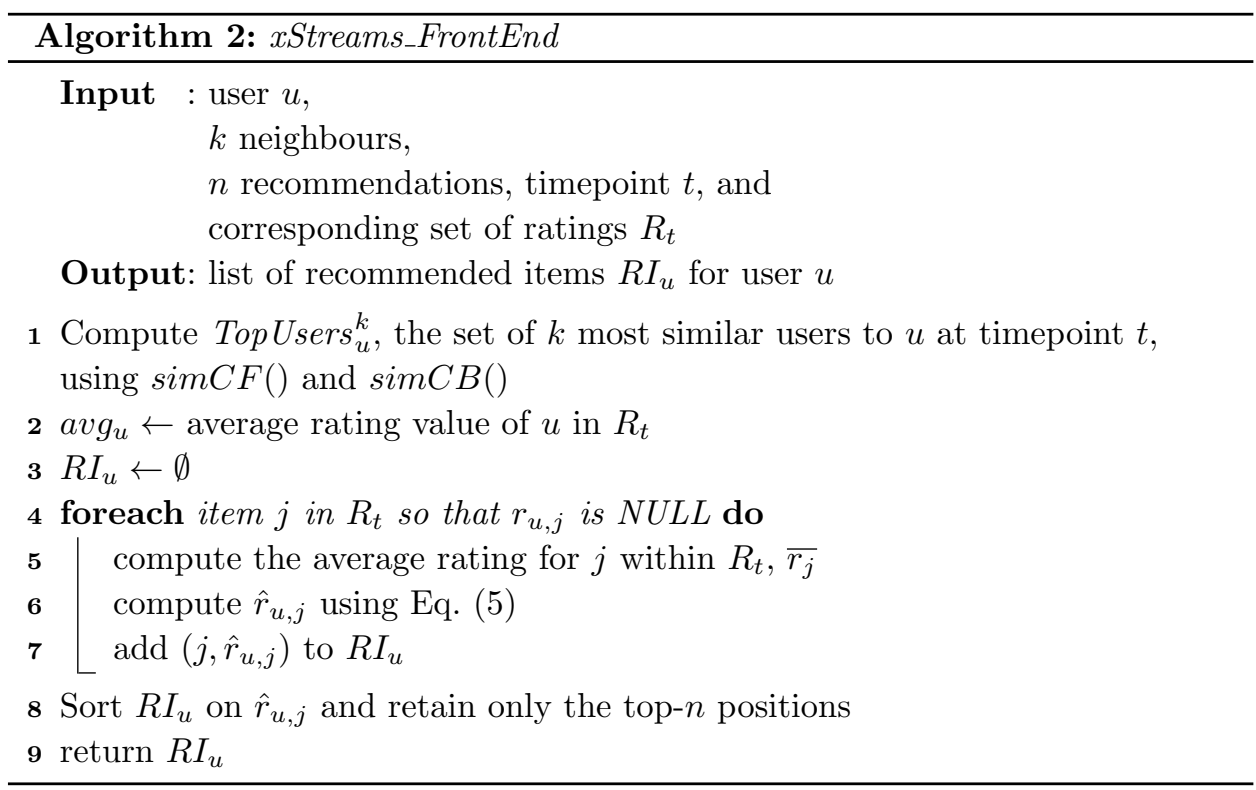

current behaviour of the users, and should concentrate on their past profiles. If the weight is less than 1 , then we use $\operatorname{sim} C F()$ to exploit also the current user behaviour, as reflected in their ratings in $R_{t}$. Using this we estimate the rating $\hat{r}_{u, j}$ that $u$ would have given to item $j$ within the current time window. Let $\overline{r_{j}}$ be the avg. rating for item $j$ in $R_{t}$, considering only items not yet rated by $u$ (line 4 ). Further, let $r_{v, j}$ be the rating that user $v \in \operatorname{Top}_{\operatorname{sers}}^{k}{ }_{u}$ has given to item $j$ within $R_{t}$. Then, the predicted $\hat{r}_{u, j}$ (line 6) is computed as shown in Eq. (5). The items with the estimated rating values are accommodated in a list (line 7 ), and the top- $n$ rated items are returned for recommendation (lines 9-10).

The items and their estimated ratings are added to set $R I_{u}$ (line 6), which is then sorted on rating value, retaining only the top- $n$ positions (line 8 ). The items at these positions are recommended to the user $u$ (line 9).

\section{MultiGen: Generating Multiple Streams of Recommendation Data}

For the evaluation of xStreams on synthetic data, we use the data generator we presented in Ref. 25, which we refer to as MultiGen hereafter. This generator creates a multi-relational stream of users, items and ratings of the users on the items, and imputes drift to it.

As we explain in Ref. 25, the generator creates three streams: the stream of Users, which is also the Target Stream, the stream of Rankings and the stream of Items (perennial) feed the target stream. The preference of a user towards some item(s) defines its behaviour. Multiple users' exhibiting similar behaviour can be grouped/categorised together as a single user profile. Conversely to learning task 


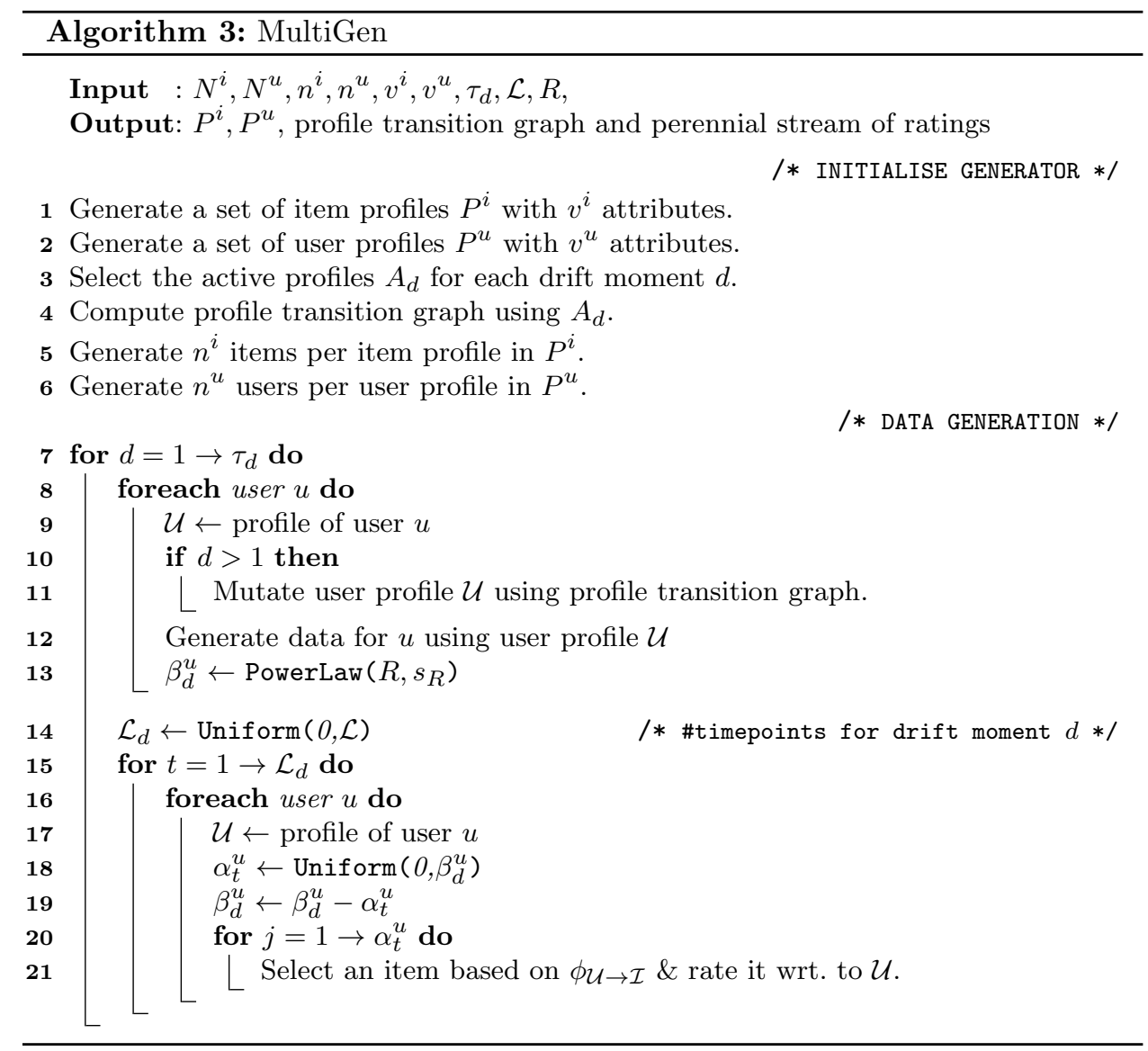

where these profiles are learned from among a group of users, MultiGen first creates these user profiles which serve as prototypes. These profiles are then used to generate individual user data according to the item preferences stored in them. Noise can be imputed to the data by forcing a user to rank in discordance to her profile with some probability. Drift is imputed to the data by allowing a profile to exist only for some timepoints and then forcing it to mutate to one or more profiles with some probability.

The complete algorithm for generating a perennial stream of ratings is given in Algorithm 3. The algorithm starts by creating a set of user profiles $P^{u}$ and items profiles $P^{i}$ (Lines 1-2). It then computes the set active user profiles $A_{d}$ for each drift moment $d$ (Line 3 ). The computation involves distributing the user profiles equally across all drift moments. Using $A_{d}$, the algorithms computes the profiles transition graph using the active profiles from consecutive drift moments, i.e. between $A_{d-1}$ and $A_{d}$ (Line 4). The creation of $n^{u}$ users and $n^{i}$ items, concludes the initialisation of MultiGen (Lines 5-6). 
The algorithm, then iterates over the defined drift moments. At the beginning, a user $u$ is assigned a random user profile $\mathcal{U}$. For the first drift moment, it uses $\mathcal{U}$ to generate the data. For the subsequent drift moments, when the drift moment changes, the $\mathcal{U}$ gets mutated into $\mathcal{U}^{\prime}$ by the profile transition graph (Lines 10-11) and the mutated profile $\mathcal{U}^{\prime}$ is used for generating further data. Then for each user, the power law distribution is used to generate ratings at a drift moment (Line 13).

A drift moment $d$ contains multiple timepoints (Line 14) and the ratings are generated at individual time points. The number of ratings to be generated for a user $u$ at timepoint $t, \alpha_{d}^{u}$, is calculated using the uniform distribution, whose lower limit is 0 while upper limit is $\beta_{d}^{u}$ (Line 18). Once $\alpha_{d}^{u}$ is calculated, the same amount is also subtracted from $\beta_{d}^{u}$ to ensure that $u$ 's rating don't exceed more than $\beta_{d}^{u}$ items (Line 19). ${ }^{\text {e }}$ The individual ratings are generated using the specifications based on the affinities of user profile $\mathcal{U}$ towards the different item profiles.

\section{Challenges in Evaluating a Stream Recommender}

Evaluation for recommenders on timestamped data is a challenging issue. DiazAviles et al. ${ }^{4}$ define a timepoint $t_{\text {split }}$ and use all objects arriving prior to this timepoint for learning and all objects arriving afterwards for testing. This approach is based on the implicit assumption that past data are adequate to predict the whole future, i.e. no concept changes occur. However, stream mining is based on the assertion that changes do occur.

In contrast to Diaz-Aviles et al., ${ }^{4}$ Nasraoui et $a l .{ }^{15}$ partition the data into batches, where each batch stores the data from a single user-profile: the batches are presented to the recommender one after the other, thus enforcing a concept shift at the end of each batch. However, Nasraoui et al. ${ }^{15}$ cater for concept change in their evaluation, but only for one particular change. For a proper evaluation, we need a less heuristic approach.

In this section we discuss some issues that are critical in order to successfully evaluate a recommender over data streams. We discuss the conventional stream evaluation, some works on streaming evaluation for recommenders and juxtapose them with the challenges that arise in evaluating a recommenders on streams.

Item recommendation can be modelled as classification problem. Stream classifiers are evaluated using hold-out evaluation or prequential evaluation. ${ }^{6}$ In hold-out evaluation, a subset of the newly arriving objects is reserved for evaluation. This approach has the disadvantage of completely wasting the information carried by the held-out objects. In prequential evaluation, new objects are first labelled by the model, so that the quality of the model is evaluated; the objects with their true labels are then used for model learning. We study whether prequential evaluation can be used for stream recommenders and devise an appropriate evaluation plan.

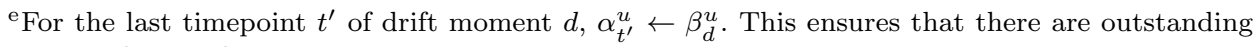
items to be rated. 


\subsection{Prequential evaluation?}

In Fig. 2, we depict the interplay of learning and evaluation for a stream recommender. At each timepoint, the recommender's model is updated by incorporating the new batch of active ratings, while the batch of old ratings is forgotten. Prequential evaluation is done on future ratings. The evaluation is subject to following challenges.

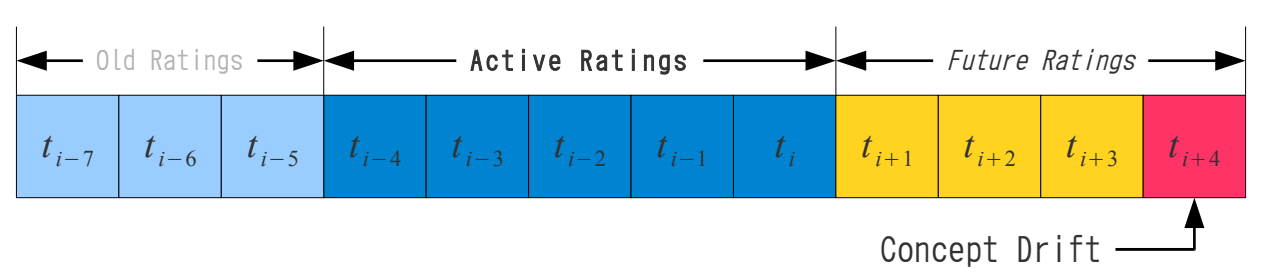

Fig. 2. Data exploitation in a stream recommender: learning is done on the active ratings (those in the window), evaluation on the future ratings, which are gradually incorporated to the sliding window and used for learning.

Challenge 1 - the span of the future: In conventional prequential evaluation, all objects to be labelled appear in the very next batch, i.e. at timepoint $t_{i+1}$. In recommenders, the users in the current batch may not appear in the batch of $t_{i+1}$. For example, assume that the recommender suggests item $x$ to user $u$. $u$ indeed rates $x$ favourably, but does so at $t^{\prime} \gg t_{i+1}$. Prequential evaluation will count a miss. A naive way to alleviate this caveat is to consider all future ratings for evaluation. This leads to the second challenge.

Challenge 2 - the drift in the future: Assume that we evaluate on all future ratings. At timepoint $t_{i+1}$, let item $x$ be recommended to user $u$, who rates it negatively at $t_{i+4}$ (a miss). At $t_{i+2}$ the model adapts to drift and learns that $u$ would dislike everything like $x$. So, after $t_{i+2}$, the model would not recommend $x$ to $u$, yet the recommendation is already done and counted. Hence, we must limit the horizon of the prequential evaluation to a window of predefined size.

Challenge 3 - the one-time users: In social and commercial sites, many users appear rarely or even only once. In the MovieLens dataset (Section 6.1), 300 users (ca. $14 \%$ of all users) appear only once. Prequential evaluation demands a recommendation for each user seen at $t_{i}$, but the outcome cannot be verified since some users might not show up again.

Challenge 4 - the casual users: Many users re-appear irregularly at timepoints that are far apart. When we discretized the MovieLens dataset into 140 months, we identified 400 users (ca. 19\%) who appear at less than 5 timepoints that are very far apart. If we use an evaluation horizon that ends before the user's next re-appearance, then prequential evaluation cannot categorize the outcome as hit or miss (similarly to Challenge 3). If we specify a huge evaluation horizon to capture the next re-appearance of such users, then we would provoke again Challenge 2. 


\subsection{Prequential evaluation with hold-outs}

In the light of the above challenges, we propose a hybrid method that sets apart a split test portion of the ratings in each incoming batch for hold-out evaluation, and performs prequential evaluation on the remaining ratings. In particular, if split $_{\text {test }}>0$, then we use $1-$ split $_{\text {test }}$ of the ratings first for evaluation and then for learning (i.e. for prequential evaluation), and split test only for evaluation. If split $_{\text {test }}=0$, we use all data for prequential evaluation only. Based on preliminary experiments, we have used split $t_{\text {test }}=0.5$.

\section{Experimental Evaluation}

We use the evaluation plan proposed in subsection 5.2 to study the performance of our method. We compare xStreams to a stream-based extension of the collaborative filtering (CF) algorithm of Ref. 26, which we call CFStream. Since we evaluate on recommendations to users, we adjust the conventional evaluation measures for recommenders as follows: for a test user who receives a list of $n$ recommended items, Precision is the ratio of the hits (favourably rated items) among the $n$ ones; Recall is the ratio of hits from the top- $n$ list to the complete set of items rated favourably by the user; RMSE is the Root Mean Square Error between predicted and true rating for the test user. Additionally, we also carry out the evaluation for MultiGen to determine how well it can simulate the distributions from the real data.

\subsection{Synthetic and real datasets}

\subsubsection{Synthetic dataset}

To study how xStreams responds to concept changes, we generated a synthetic dataset with predefined moments of drift. We use the MultiGen to create streams of users, items and ratings. The schema of the generated relational data is shown in Figure 3(a).

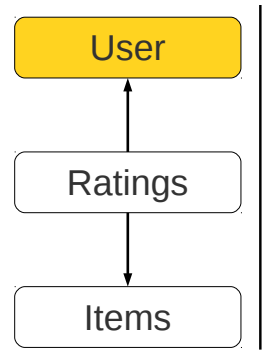

(a)

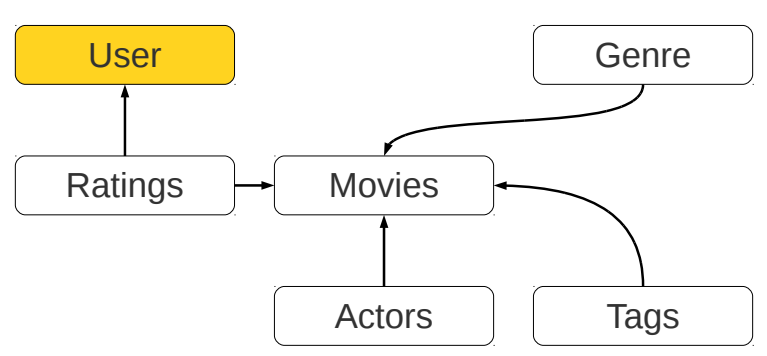

(b)

Fig. 3. Schema of (a) synthetic dataset, and (b) MovieLens dataset. 


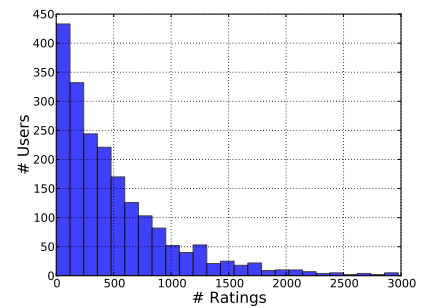

(a)

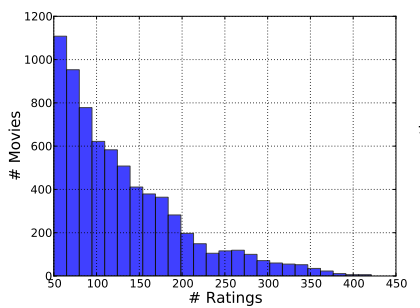

(b)

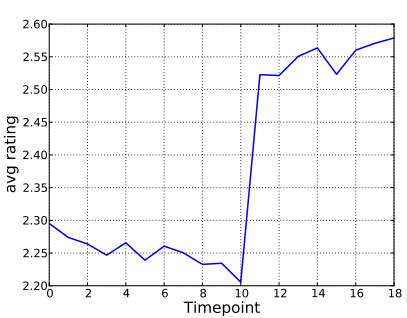

(c)

Fig. 4. Statistics for synthetic dataset: (a) number of ratings vs. user, (b) number of ratings vs. movies, (c) average rating value vs. timepoint.

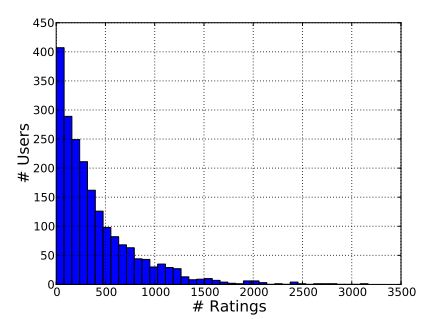

(a)

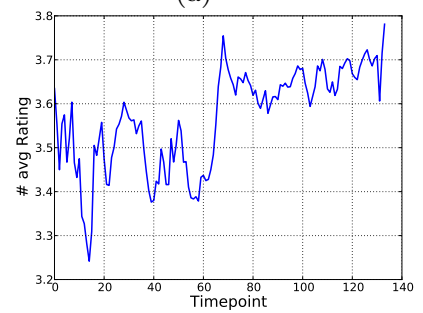

(d)

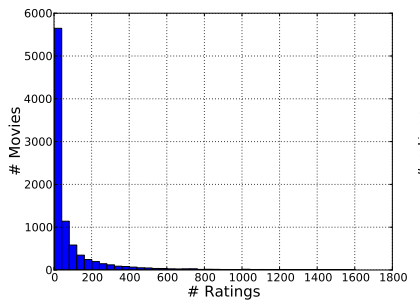

(b)

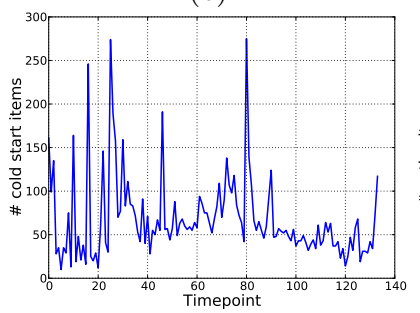

(e)

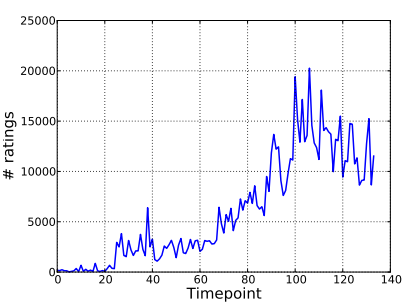

(c)

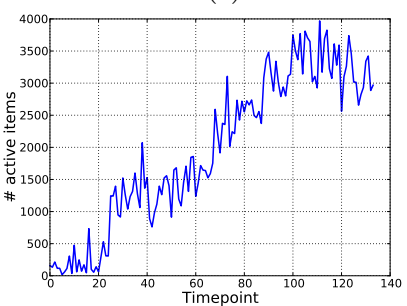

(f)

Fig. 5. Statistics for MovieLens, recorded at each timepoint: from top: l-to-r, (a) \#ratings per user, (b) \#ratings per movie, and for each timepoint (c) \#ratings, (d) avg. rating, (e) \# cold start items and (f) \# rated items.

MultiGen is capable of simulating the properties of real world datasets as shown in Figures 4(a) and 4(b), which they both follow the Zipf distribution similar to the results shown in Figures 5(a) and 5(b), respectively. Moreover, in Figure 4(c) we have incorporated explicit concept drift into our synthetic data. It is a shift of average rating that happens around timepoint 8 , where the mean rating value jumps from around 2.25 stars to 2.55 stars. This concept drift forces the users to change their rating behaviour abruptly around timepoint 8 . As will be later experimentally shown, our xStreams algorithm is able to adapt fast to the concept drift.

This generator simulates the properties of datasets like MovieLens: users, items and ratings follow power law distributions and over time, users change their rating preferences. 


\subsubsection{Real dataset}

We have used MovieLens ${ }^{\mathrm{f}}$ dataset. It contains 2113 users, 10,197 movies and approximately 0.85 million ratings. Additionally to the user-item rating matrix, the dataset includes information on each movie's genre, directors, actors and user tags. This auxiliary information is provided as separate streams/tables. We have created a multi-stream by using the schema of Figure 3(b), whereby we grouped actors into 6 categories, by ranking them on importance of their role in a movie multiplied by the average rating of those movie. Summing up these values over all the movies an actor was involved gives us an AScore for an actor:

$$
A \operatorname{Score}(a)=\sum_{m \in \operatorname{movies}(a)}(250-\operatorname{Rank}(a, m)) * M \operatorname{Score}(m) .
$$

The six categories are based on AScore where, C1 accommodates first 18 actors, C2 the next 50, C3 the next 200, C4 the next 500, C5 the next 5000 and C6 all the rest.

In Figure 5 we show statistics on MovieLens. MovieLens dataset follows zipf distribution for both the number of ratings provided by users and the number of ratings given to a particular movie, i.e. there is a small number of users who have rated many items (short head) and many users that have only rated a small number of items (long tail). There are only few ratings before $t_{30}$ (Figure $5(\mathrm{c})$ ). The bulk of rating starts arriving after $t_{70}$. The average rating value increases continuously with a sharp increase (concept shift) at $t_{80}$ (Figure $5(\mathrm{~d})$ ). Around $t_{80}$, there also is a large influx of new cold-start items (Figure 5(e)). We show later that the concept shifts and the cold-start items affect the performance negatively, while the increase in \#ratings and of rated items have a positive effect.

\subsection{Experiments with MultiGen}

In this section, we show that MultiGen is capable of simulating the properties of a real world dataset, namely the dataset hetrec2011-movielens-2k described below. In this section we provide the empirical analysis of our generator. ${ }^{\mathrm{g}}$ In the experiments, we first vary the main parameter $s$ of the distribution (skewness parameter) by keeping $v=1$ (cf. Ref. 25, Table of parameters) and we vary the parameter $v$ and the maximum number items rated by a user, by keeping the value of $s$ equal to 3 .

\subsubsection{Quantitative results}

Datasets from the MovieLens are widely used for testing of the algorithms. From the various datasets available at the GroupLens web site, ${ }^{\mathrm{h}}$ we chose hetrec2011-

\footnotetext{
${ }^{\mathrm{f}}$ GroupLens website: http://grouplens.org/datasets/hetrec-2011/; Dataset: hetrec2011-movielens$2 \mathrm{k} . z i p$

${ }^{\mathrm{g}}$ The data generator along with further results can be downloaded from http://www.kmd.ovgu.de/ kmd_media/Downloads/Software/2014_MultiGen_generator.zip

h http://www.grouplens.org/node/73
} 
movielens-2k. It has 2113 users, approximately 10,000 movies and approximately 0.85 million ratings provided by the users. Additionally with the dataset the information about each movie, i.e. genre, cast, location is also provided. We treat this as our baseline and compare the results of MultiGen against it.

\subsubsection{Validation measures}

Common measures used for parameter calibrations and validation are the Mean Absolute Error (MAE) and the Root Mean Square Deviation (RMSD). Especially $R M S D$ is a good measure of the accuracy. ${ }^{8}$

For two separate samples, both of cardinality $N: A=\left\{a_{1}, \ldots, a_{N}\right\}, B=$ $\left\{b_{1}, \ldots, b_{N}\right\}$, MAE and RMSD are defined as follows:

$$
M A E=\frac{1}{n} \sum_{i=1}^{n}\left|a_{i}-b_{i}\right|, \quad R M S D=\sqrt{\frac{1}{n} \sum_{i=1}^{n}\left(a_{i}-b_{i}\right)^{2}} .
$$

We have $N=2113$ users, $a_{i}$ is the number of ratings in hetrec2011-movielens$\mathbf{2 k}$ for the user $i,(i=1, \ldots, n)$, and $b_{i}$ are the corresponding number of ratings for the user $i$ in the generated dataset.

\subsubsection{Results}

We found experimentally that the best fit holds when $s=21.5, v=10000, R=$ 3000. In the Figure 6 we compare the distributions from the MovieLens dataset and the dataset generated by MultiGen. The discovered parameter settings minimize both errors: $M A E=105.13, R M S D=142.57$. However, these parameter settings can still be further improved as seen in the figure, especially in terms of which items get rated more and how much (see left of Figure 6). The skewed effect on the items rating is more clearly visible in Figure 7 . Currently, we use random variable for generating the number of ratings by a customer only and the same random number
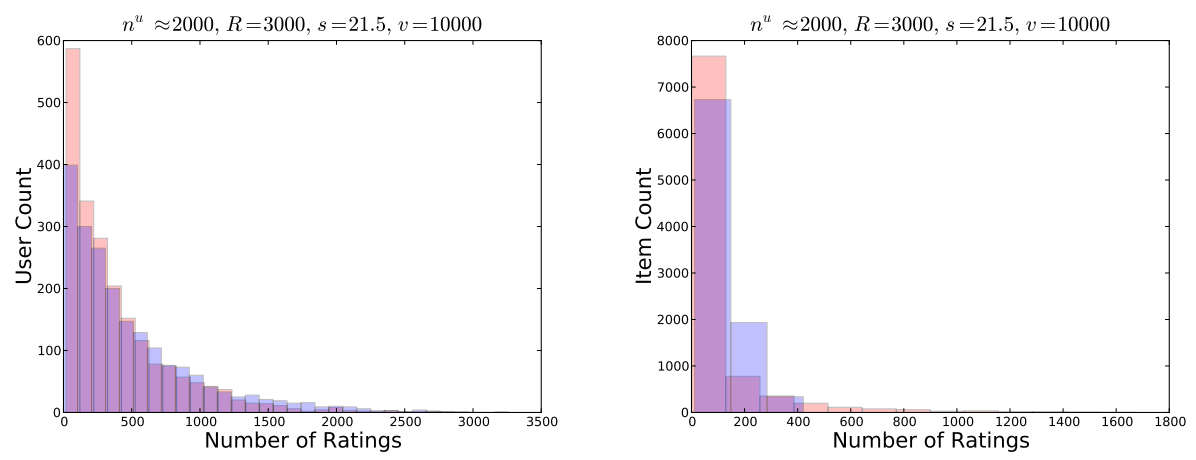

Fig. 6. (Color online) Comparison of the dataset from MultiGen and hetrec2011-movielens$\mathbf{2 k}$ dataset. (Left) distribution of the number of ratings provided the user (right) number of ratings an item has. 

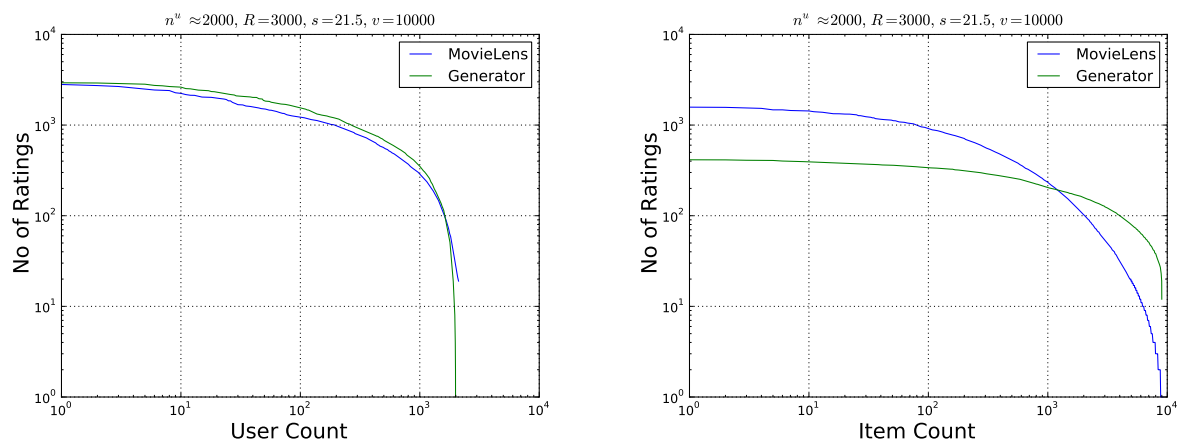

Fig. 7. (Color online) Log-Log plot from Figure 6.

is used implicitly for selecting an item. For this reason, it is easier to predict the effect of parametric changes on the number of ratings by the customers but not on the items.

In Figure 7 we see that the distributions are similar, but plot for users in MovieLens (on the left) one ends abruptly. This is because the data provider has removed all the users who have less than 20 ratings. This made it slightly difficult to estimate the correct parameter for the Zipf distribution.

In Figures 8-11 we show the effects of parametric change on how the ratings for the items get affected.

\subsection{Experiments on synthetic data}

We first study the performance of xStreams for various sizes of the sliding window: $w=2,4,8$. We set xStreams to return the top- $n$ recommendations for $n=7$ and to consider the $k=7$ nearest neighbors (cf. Algorithm 2). We use only $\operatorname{sim} C B($ ), i.e. set weight $=1.0$ in Eq. (5) and split test $=0.0$.

In Figures 12(a) and (b), we see that the smallest window size $w=2$ achieves the best RMSE curve: the values are low and the recovery after the concept shift at $t_{8}$ is fast. This indicates that forgetting old data soon is best if the data exhibit drifts. We have also run this experiment for Precision, Recall and F-Measure and also for $k=30$; we observed the same trend.

In Figures 12(c) and (d), we compare xStreams to CFStream. For xStreams, settings are same except the window is set to $w=2$ and varying the weight of $\operatorname{sim} C B()$ in Eq. (5), assigning the values 1.0, 0.67, 0.34. xStreams outperforms CFStream and exhibits lowest RMSE when the weight of $\operatorname{sim} C B()$ in the ratings is 0.67 ; this means that both the similarity of users on accumulated past data, as captured by $\operatorname{sim} C B()$, and the conventional similarity on the ratings should be taken into account. However, CFStream that only considers the conventional similarity has lower performance. When evaluating on F-measure, Precision and Recall (not shown), xStreams also outperformed CFStream; for Precision and Recall, the 

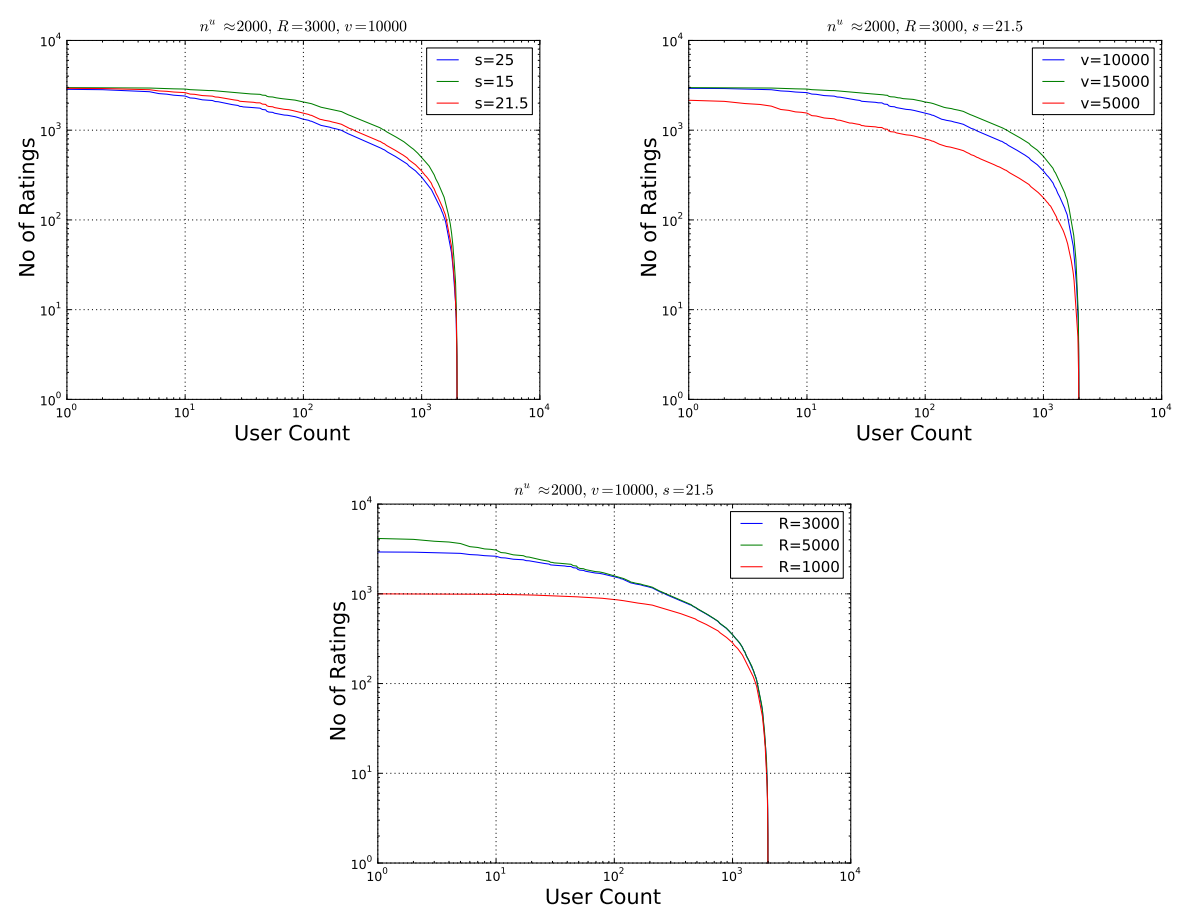

Fig. 8. (Color online) Effect of changing various parameters of the Zipf distribution on the user's rating behaviour (left) variable $s$, (right) variable $v$ and (bottom) variable $R$.

values for weight $=1.0$ were better than for weight $=0.67$ in the first half of the timepoints but deteriorated thereafter; for weight $=0.67$, the performance was more stable.

\subsection{Experiments on real data}

We evaluate xStreams to CFStream on MovieLens. Over the 140 timepoints (months) of the MovieLens data, we slide a window $w$ of length 12 .

We show the curves on Precision and RMSE for $w=12, n=2$, and $k=100$ in Figures 13(a) and (b), respectively. The evaluation measures illustrate nicely how different parameter settings respond to these two counteracting measures. All the strategies had a low recall, which was around 0.04 with little variance among, therefore we omit their graphs. The algorithm allows for the exploitation of more past data because of the larger window size $(w=12)$. We have set the number of neighbours $k=100$ and used a split test $=0.5$, i.e. half of the arriving ratings are held-out for evaluation. We start with the delivery of recommendations at $t_{30}$, because the numbers of ratings and rated items are very low in the first timepoints, while the number of cold-start items is very high (see Figures 5(a), (d) and (c), respectively). 

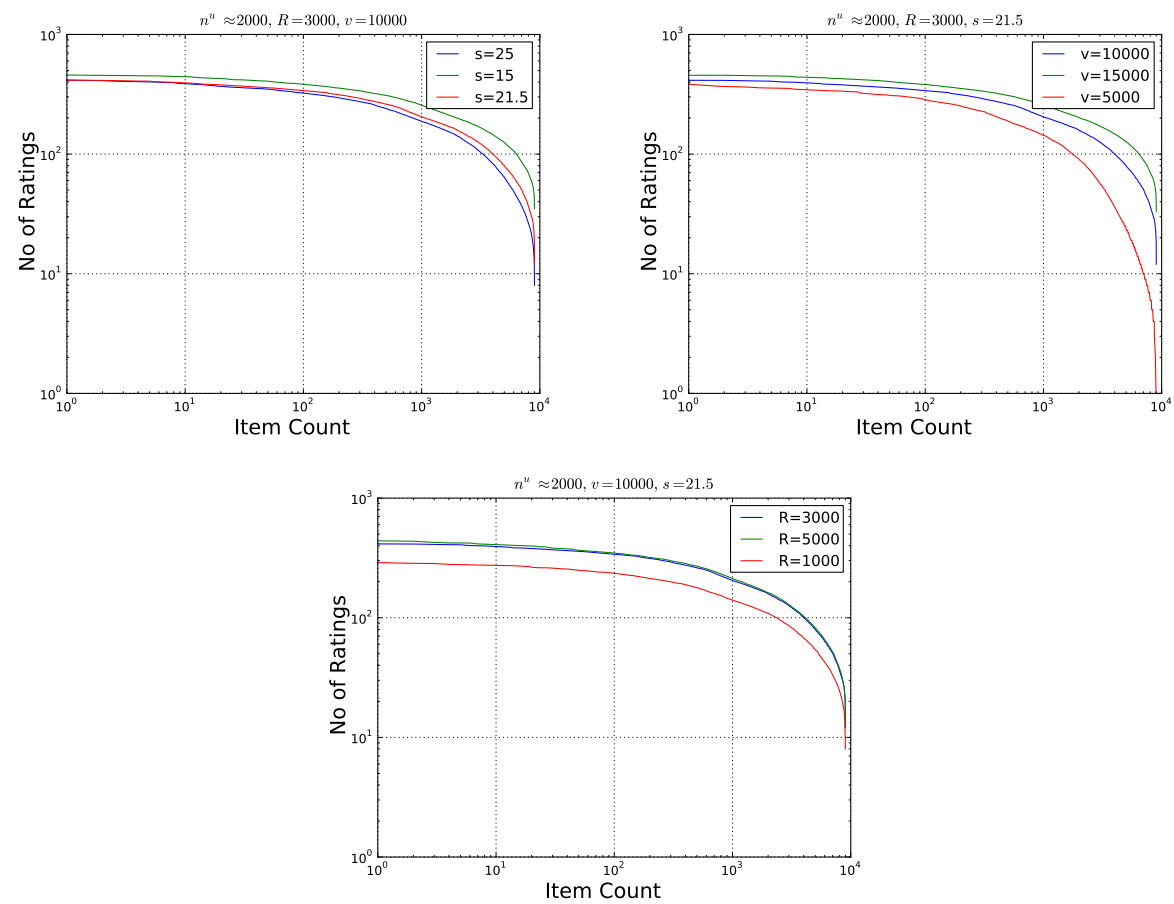

Fig. 9. (Color online) Effect of changing various parameters of the Zipf distribution on the how items get rated (left) variable $s$, (right) variable $v$ and (bottom) variable $R$.
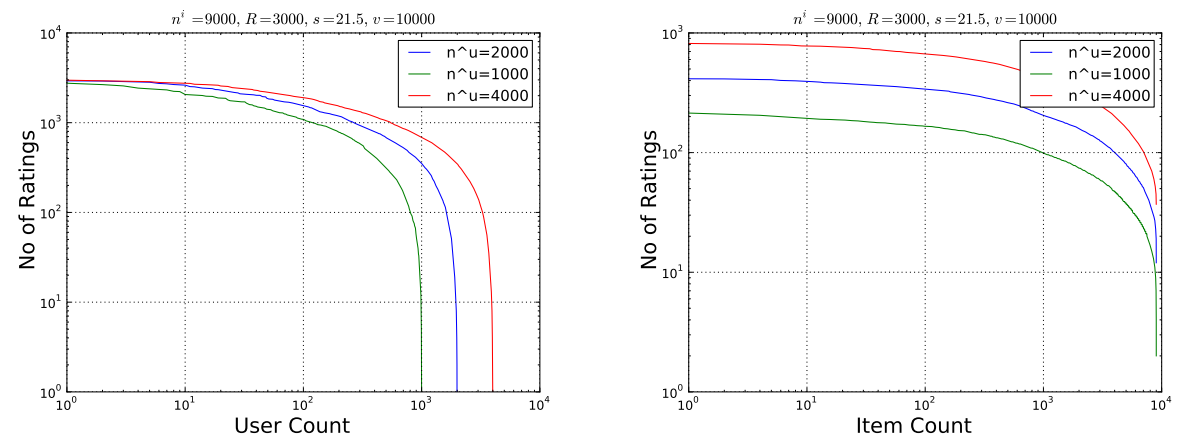

Fig. 10. (Color online) Effect of changing the parameter $n^{u}$ on the ratings' distribution for user and item.

It is obvious that precision (and also recall) values depend strongly on the number of recommendations. Thus, if we increase the $n$ parameter, precision will fall (and recall will increase). Comparing the curves for the two $n$ values, we observe that the setting $n=2$ and $n=7$ (see Figures 13(d) and (d)) leads to lower performance. Returning the top- 2 recommendations is more challenging, but we expected that 

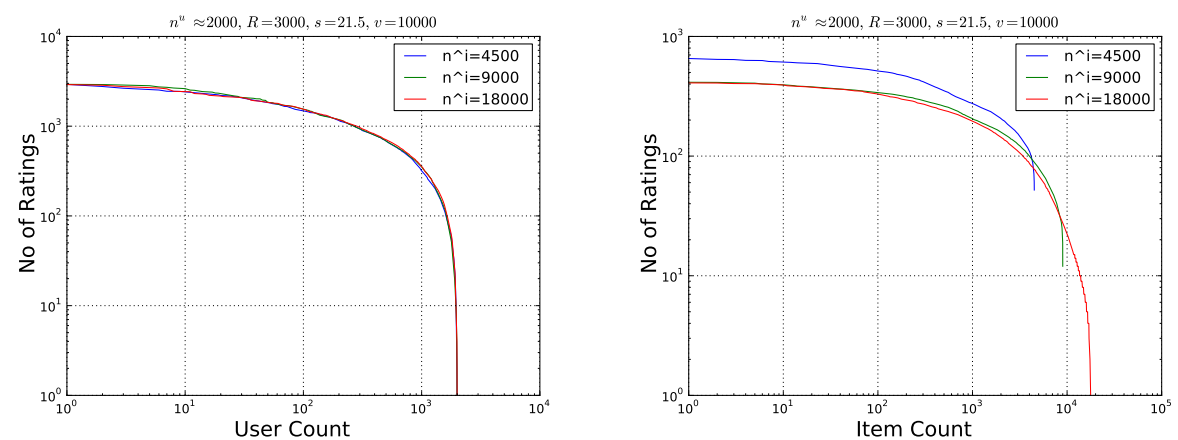

Fig. 11. (Color online) Effect of changing the parameter $n^{i}$ on the no of ratings distribution for user and item.

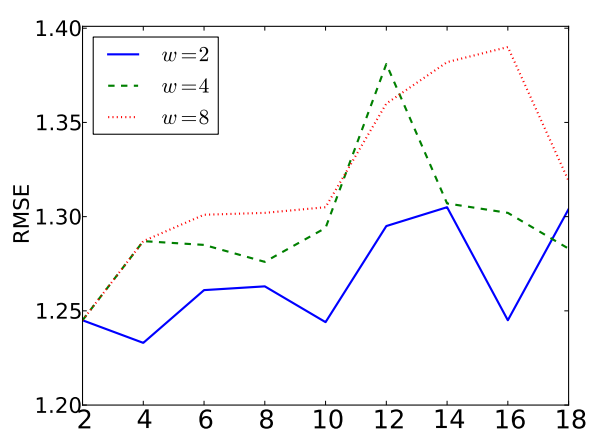

(a)

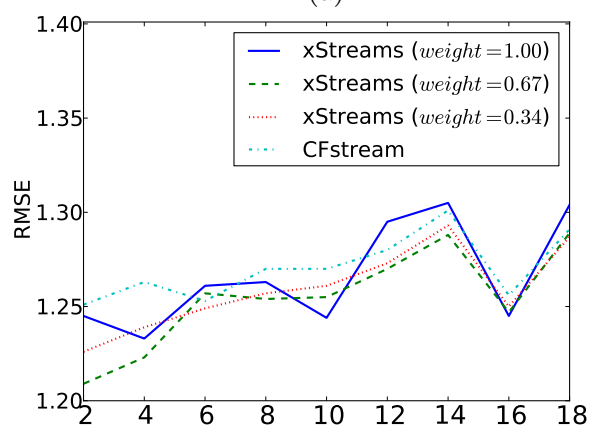

(c)

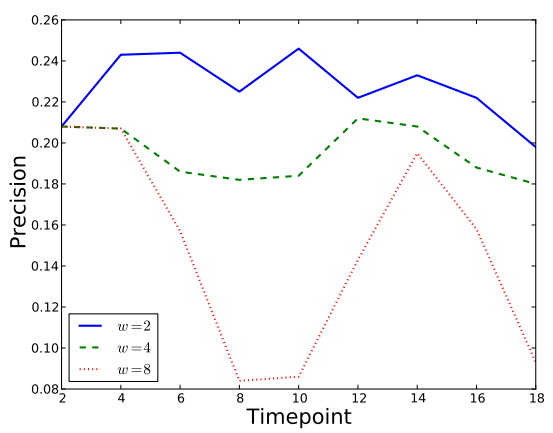

(b)

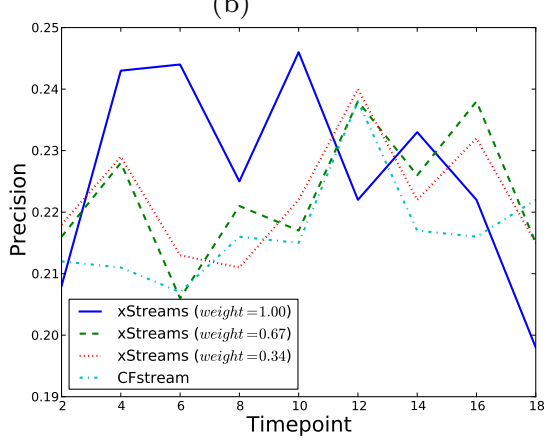

(d)

Fig. 12. (Color online) RMSE values for xStreams: (a) and (b) under different windows sizes $w=2,4,8(\operatorname{sim} C B()=1.0) ;(\mathrm{c})$ and $(\mathrm{d})$ when comparing xStreams to CFStream $(w=2)$, for $k=7$ nearest neighbours and returning $n=7$ recommendations; lower values are better.

the large window size and the use of $\operatorname{sim} C B()$ would have partially compensated it. The precision drops faster for $n=7$ (Figure 13(a) vs. (c)). A further reason for the low performance is the use of split test $=0.5$ : setting aside half of the data for evaluation has a stronger negative impact in the larger window. 


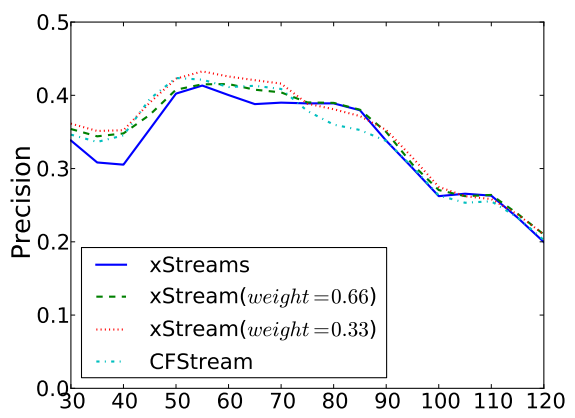

(a)

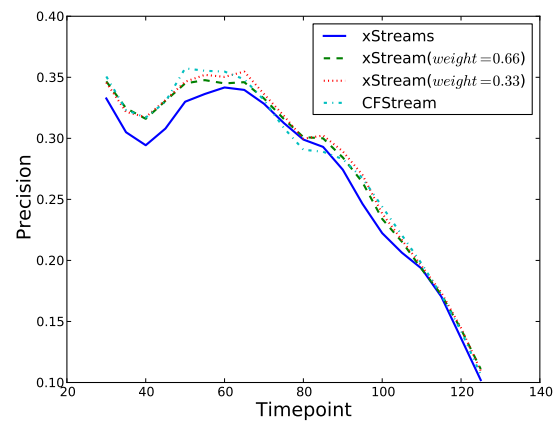

(c)

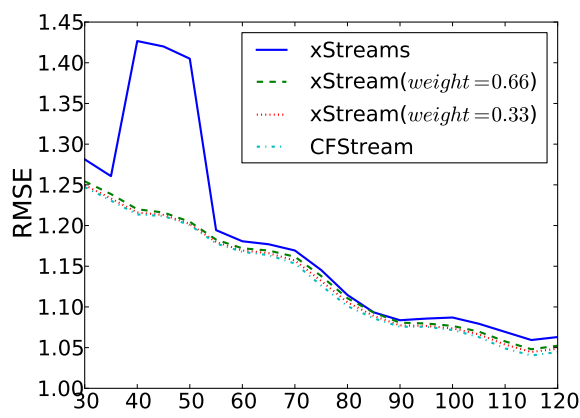

(b)

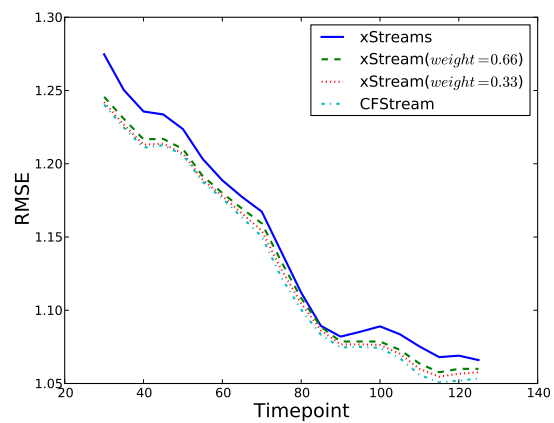

(d)

Fig. 13. (Color online) Comparison of xSreams \& CFStream: (a) Precision and (b) RMSE for $n=2, w=12, k=100$; (c) Precision and (d) RMSE for $n=7, w=12, k=100$.

Among the different variants of xStream and the CFStream there is no clear winner. Rather, each strategy outperforms the others at different moments. CFStream performs comparably to xStreams in the first moments, while the variants of xStreams with a weight of $67 \%$ or $0.33 \%$ for $\operatorname{sim} C B()$ show consistently better performance after $t 60$, i.e. they cope well with the influx of cold start items at $t 80$ (Figure 5(c)). Since CFStream corresponds to a weight of 0 , and since xStreams with weight $=1.0$ shows lower precision/recall values, we conclude that it is necessary to combine the similarity among recent ratings (done by $\operatorname{sim} C F()$ ) with the similarity of the profiles incorporating the users' past behaviour (as done by $\operatorname{sim} C B())$.

\section{Conclusion}

\subsection{Summary}

We have presented a stream-based recommendation method that learns and adapts to the user preferences, as these preferences evolve over time. Preferences are reflected in the ratings that users give to items; we accumulate them to user profiles, 
and use stream clustering to build the profiles and adjust them to change. This gives the basis for a more elaborate notion of similarity: instead of a static similarity between users, we have a dynamic similarity between user profiles. To alleviate possible negative effects caused by very large profiles (sparse clusters with large radius), we also restrict similarity further by requiring proximity within the profile. Hence, two users are similar at some moment, if they have the same profile at this moment and are proximal within the profile.

We have also presented a multi-stream generator that has been inspired from the domain of recommendation system. It generates ratings data for users according to user profiles. With time the profiles mutates into newer ones. The mutation can be adjusted to simulate drastic shifts as well more gradual drifts. The generator can be used for evaluating supervised and unsupervised learning task for discovering and adaptation to concept drift.

We have studied our approach on a synthetic and a real dataset. The synthetic dataset has been used to depict the effects of change in a controlled, transparent way. For this dataset, we have shown that our method experiences a performance deterioration after the imputed change, whereupon it replaces gradually but swiftly the outdated profiles with new ones, and recovers fast. The real dataset MovieLens exhibits less drastic forms of change, whereupon our approach also shows smoother performance. Strategies with larger window size achieve higher precision and average RMSE (which is apparently dominated by precision) at the cost of low recall. The best balance between precision and recall is achieved by a strategy with small window size, i.e. one that maintains little profile information and replaces the profiles as soon as performance deteriorates.

\subsection{Discussion and future work}

An intriguing finding was the discrepancy between precision and recall for all strategies, i.e. for both our stream-based strategies with different window sizes and for the change-insensitive baseline. A possible explanation, which requires further investigation though, is the power law that governs the data distribution. The long tail shown in Figure 2 implies that little information is available for most of the users: perhaps, high precision is achieved by learning a lot about the few users in the short head, while the low recall is caused by the long tail. This seems to be supported by the fact that best recall is achieved by the strategy with the smallest window size, which exploits little information about the users and forgets learned profiles fast. A deeper study of this issue is our next planed task.

Related to the above is the future study of the implications of power law on the similarity among users: there are many users with many ratings, but most users have few ratings on mostly different items. This makes the computation of similarity more complicated. We want to investigate elaborate similarity measures for such users. This will allow us to tackle the problem of formulating recommendations for the many users in the long tail, whose preferences evolve no less than those of the few users in the short head. 
In multi-stream generator, we allow the user profiles to mutate over time, however, the items remain static. In real world the item profiles under go change as well, e.g. comedy movies have gradually gotten more and more anarchic or with the advances in technology the sci-fi genre have gradually changed its outlook. While the mutation of user profiles represents sudden shift in the distribution of the data, by incorporating the dynamics of change for item profiles, more gradual shift can be incorporated into the dataset. Such a generator would also be beneficial trajectory-based stream methods.

\section{Acknowledgments}

Part of this work was funded by the German Research Foundation project SP 572/11-1 "IMPRINT: Incremental Mining for Perennial Objects" (first author), and by the Greek Scholarship Foundation (IKY) for postdoctoral studies (ESPA 2007-2013) (third author).

\section{References}

1. M. Balabanovic and Y. Shoham, Fab: Content-based, collaborative recommendation, ACM Communications 40(3) (1997) 66-72.

2. M. Deshpande and G. Karypis, Item-based top- $N$ recommendation algorithms, $A C M$ Transactions on Information Systems 22(1) (2004) 143-177.

3. M. B. Dias, D. Locher, M. Li, W. El-Deredy and P. J. Lisboa, The value of personalised recommender systems to e-business: A case study, in Proc. of the 2nd ACM Conference on Recommender Systems (RecSys 2008) (2008), pp. 291-294.

4. E. Diaz-Aviles, L. Drumond, L. Schmidt-Thieme and W. Nejdl, Real-time top-N recommendation in social streams, in Proc. of the 6th ACM Conference on Recommender systems (RecSys 2012) (2012).

5. Y. Ding and X. Li, Time weight collaborative filtering, in Proc. of the 14th ACM Int. Conf. on Information and Knowledge Management (CIKM 2005) (2005), pp. 485-492.

6. J. Gama, R. Sebastião and P. P. Rodrigues, Issues in evaluation of stream learning algorithms, in Proc. 15th ACM Int. Conf. on Knowledge Discovery and Data Mining (KDD 2009) (ACM, 2009), pp. 329-338.

7. J. Herlocker, J. A. Konstan and J. Riedl, An empirical analysis of design choices in neighborhood-based collaborative filtering algorithms, Information Retrieval 5(4) (Oct. 2002) 287-310.

8. J. L. Herlocker, J. A. Konstan, L. G. Terveen and J. T. Riedl, Evaluating collaborative filtering recommender systems, ACM Trans. Inf. Syst. 22(1) (Jan. 2004) 5-53.

9. Y. Koren, Collaborative filtering with temporal dynamics, in Proc. 15th ACM Int. Conf. on Knowledge Discovery and Data Mining (KDD 2009) (2009), pp. 447-456.

10. Y. Koren, Collaborative filtering with temporal dynamics, Communications of ACM 53(4) (2010) 89-97.

11. Y. Koren and R. Bell, Advances in collaborative filtering, in Recommender Systems Handbook, eds. F. Ricci, L. Rokach, B. Shapira and P. B. Kantor, Chap. 5 (Springer Science+Business Media, 2011), pp. 145-186.

12. M. R. McLaughlin and J. L. Herlocker, A collaborative filtering algorithm and evaluation metric that accurately model the user experience, in Proc. of the ${ }^{2}{ }^{7}$ th ACM Int. 
Conf. on Research and Development in Information Retrieval (SIGIR 2004) (ACM, 2004), pp. 329-336.

13. R. J. Mooney and L. Roy, Content-based book recommending using learning for text categorization, in Proc. of the 5th ACM Conference on Digital Libraries (CDL 2000) (ACM, 2000), pp. 195-204.

14. O. Nasraoui, C. Cardona-Uribe and C. Rojas-Coronel, Tecno-Streams: Tracking evolving clusters in noisy data streams with an scalable immune system learning method, in Proc. of the 3rd IEEE Int. Conf. on Data Mining (ICDM 2003) (Melbourne, Australia, 2003).

15. O. Nasraoui, J. Cerwinske, C. Rojas and F. Gonzalez, Performance of recommendation systems in dynamic streaming environments, in Proc. of the SIAM Int. Conf. on Data Mining (SDM 2007) (2007).

16. A. Papadimitriou, P. Symeonidis and Y. Manolopoulos, A generalized taxonomy of explanation styles for traditional and social recommender systems, Data Mining and Knowledge Discovery 24(3) (2012) 555-583.

17. M. J. Pazzani and D. Billsus, Learning and revising user profiles: The identification of interesting web sites, $M L \mathbf{2 7}(3)$ (1997) 313-331.

18. A. Prieditis and S. J. Russell (eds.), Machine Learning, Proc. of the Twelfth Int. Conf. on Machine Learning (Morgan Kaufmann, 1995) (Tahoe City, California, USA, July 9-12, 1995).

19. P. Resnick, N. Iacovou, M. Suchak, P. Bergstrom and J. Riedl, GroupLens: An open architecture for collaborative filtering on netnews, in Proc. of the Computer Supported Collaborative Work Conference (RISBR 1994) (1994), pp. 175-186.

20. J. Salter and N. Antonopoulos, CinemaScreen recommender agent: Combining collaborative and content-based filtering, Intelligent Systems Magazine 21(1) (2006) 35-41.

21. B. Sarwar, G. Karypis, J. Konstan and J. Riedl, Item-based collaborative filtering recommendation algorithms, in Proc. of the 10th International World Wide Web Conference ( $W W W$ 2001) (2001), pp. 285-295.

22. Z. F. Siddiqui and M. Spiliopoulou, Combining multiple interrelated streams for incremental clustering, in Proc. of the 21st Int. Conf. on Scientific and Statistical Database Management (SSDBM 2009) (2009).

23. Z. F. Siddiqui and M. Spiliopoulou, Stream clustering of growing objects, in Proc. of 12th Int. Conf. on Discovery Science (DS 2009) (2009).

24. Z. F. Siddiqui and M. Spiliopoulou, Tree induction over perennial objects, in Proc. of the 22nd Int. Conf. on Scientific and Statistical Database Management (SSDBM 2010) (Springer-Verlag, 2010), pp. 640-657.

25. Z. F. Siddiqui, M. Spiliopoulou, P. Symeonidis and E. Tiakas, A data generator for multi-stream data, in Proc. of the 2nd Int. Workshop on Mining Ubiquitous and Social Environments (MUSE 2011) (at the ECML PKDD 2011, Athens, Greece, September 5, 2011), pp. 70-78 of the document. http://www.kde.cs.unikassel.de/ws/muse2011/proceedings.pdf

26. P. Symeonidis, E. Tiakas and Y. Manolopoulos, Product recommendation and rating prediction based on multi-modal social networks, in Proc. of the 5th ACM Conference on Recommender Systems (RecSys 2011) (2011), pp. 61-68.

27. L. Xiang, Q. Yuan, S. Zhao, L. Chen, X. Zhang, Q. Yang and J. Sun, Temporal recommendation on graphs via long- and short-term preference fusion, in Proc. 16th ACM Int. Conf. on Knowledge Discovery and Data Mining (KDD 2010) (Washington, DC, 2010), pp. 723-732.

28. G. Xue, C. Lin, Q. Yang, W. Xi, H. Zeng, Y. Yu and Z. Chen, Scalable collaborative filtering using cluster-based smoothing, in Proc. of the 28th ACM Int. Conf. on 
Research and Development in Information Retrieval (SIGIR 2005) (Salvador, Brazil, 2005), pp. 114-121.

29. Q. Yuan, G. Cong, Z. Ma, A. Sun and N. Magnenat-Thalmann, Time-aware pointof-interest recommendation, in Proc. of the 36th ACM Int. Conf. on Research and Development in Information Retrieval (SIGIR 2013) (Dublin, Ireland, 2013), pp. 363-372. 